\title{
PASSWORD-AUTHENTICATED GROUP KEY ESTABLISHMENT FROM SMOOTH PROJECTIVE HASH FUNCTIONS
}

\author{
Jens-Matthias BOHLi ${ }^{a}$, MARÍA Is Abel GONZÁLEZ VASCO $^{b, *}$, RAINER STEINWANDT $^{c}$ \\ ${ }^{a}$ Department of Information Technology \\ Mannheim University of Applied Sciences, Paul-Wittsack-Straße 10, 68163 Manheim, Germany \\ e-mail: j.bohli@hs-mannheim.de \\ ${ }^{b}$ Department of Applied Mathematics, Materials Science and Technology, and Electronic Engineering (MACIMTE) \\ King Juan Carlos University, C/ Tulipán s/n, 28933 Móstoles, Madrid, Spain \\ e-mail: mariaisabel.vasco@urjc.es \\ ${ }^{c}$ Department of Mathematical Sciences \\ Florida Atlantic University, 777 Glades Road, Boca Raton, FL 33431, USA \\ e-mail: rsteinwa@fau.edu
}

\begin{abstract}
Password-authenticated key exchange (PAKE) protocols allow users sharing a password to agree upon a high entropy secret. Thus, they can be implemented without complex infrastructures that typically involve public keys and certificates. In this paper, a provably secure password-authenticated protocol for group key establishment in the common reference string (CRS) model is presented. While prior constructions of the group (PAKE) can be found in the literature, most of them rely on idealized assumptions, which we do not make here. Furthermore, our protocol is quite efficient, as regardless of the number of involved participants it can be implemented with only three communication rounds. We use a (by now classical) trick of Burmester and Desmedt for deriving group key exchange protocols using a two-party construction as the main building block. In our case, the two-party PAKE used as a base is a one-round protocol by Katz and Vaikuntanathan, which in turn builds upon a special kind of smooth projective hash functions (KV-SPHFs). Smooth projective hash functions (SPHFs) were first introduced by Cramer and Shoup (2002) as a valuable cryptographic primitive for deriving provable secure encryption schemes. These functions and their variants proved useful in many other scenarios. We use here as a main tool a very strong type of SPHF, introduced by Katz and Vaikuntanathan for building a one-round password based two party key exchange protocol. As evidenced by Ben Hamouda et al. (2013), KV-SPHFs can be instantiated on Cramer-Shoup ciphertexts, thus yielding very efficient (and pairing free) constructions.
\end{abstract}

Keywords: group key exchange, password authentication, smooth projective hashing.

\section{Introduction}

Key exchange protocols are among the most useful cryptographic constructions, allowing users interacting through an insecure network to establish a common secret that can later be used for achieving strong confidentiality and integrity guarantees.

In distributed applications, low-entropy passwords are still a dominating tool for authentication. Reflecting this, significant research efforts are currently devoted to the exploration of password-authenticated key

\footnotetext{
*Corresponding author
}

establishment protocols, through which participants sharing initially a short password aim at agreeing upon a high entropy secret for securing subsequent communication. In this contribution we focus on group key establishment involving $n \geq 2$ users.

In the password setting, different scenarios can be considered depending on the application context. For example, it can be plausible to assume that a dedicated server is available, and each user has an individual password shared with this server. A different scenario does not involve a server, and assumes that all users involved in the key establishment share a common 
password. In this paper we consider the latter approach, which is well suited for small user groups without a centralized server. In such scenarios, multicast message confidentiality or data integrity are natural cryptographic goals that can be achieved once the group has established a shared high-entropy secret key (this is the case, for instance, of secure virtual conferences involving up to a hundred participants). Similarly, in applications where the legitimate protocol participants are different devices controlled by a single human user, a shared high-entropy key may be used to ensure data integrity.

1.1. Key establishment: From 2-party to group. The design of key establishment protocols for two participants has been extensively studied during the last decades, both in the password setting (Boyko et al., 2000; Katz et al., 2001; Abdalla and Pointcheval, 2005; Abdalla et al., 2015) or using stronger authentication means (signatures) (Blake-Wilson and Menezes, 1999; Bellare et al., 1998; Katz and Yung, 2007). A standard strategy for breaking down the design task of a group key establishment into conceptually simpler steps are protocol compilers that build on the security of a given 2-party solution. Indeed, a number of such generic constructions have been discussed in the literature (Burmester and Desmedt, 1995; Mayer and Yung, 1999; Hwang et al., 2004). Many of these compilers are inspired by the classical construction of Burmester and Desmedt (1995), where the trick of establishing a group key from pairwise agreed keys among the group principals was first introduced. We sketch their idea in Fig. 1, where AKE stands for two-party authenticated key exchange protocol, and thus $\operatorname{AKE}(A, B)$ denotes the execution of $\mathrm{AKE}$ by users $A$ and $B$.

The Burmester-Desmedt trick goes as follows: assume participants to be arranged in a cycle. In a first round, each participant exchanges two-party keys with his left and right neighbour. Once these two-party key establishments have been completed, each participant broadcasts the XOR-value (or the quotient) of the two keys he shares with his neighbors. This allows everyone in the cycle to retrieve each of the 2-party keys that have been exchanged previously, from which a shared session key may be derived. Intuitively, if an adversary has not been able to compromise the security of any of the 2-party protocol executions involved, neither will he be able to retrieve any information about the resulting group session key (for XORs of "randomly looking" elements should look as well random to him), even though some precautions must be taken in order to prevent him from mixing up the messages exchanged in the last rounds.

1.2. Related work and our contribution. Remarkably, whether designed following the above idea or built from scratch, most group key exchange protocols that can be found in the literature rely on high-entropy secrets for achieving security against active adversaries. Using instead password-based authentication, the first such construction in the standard model is due to Abdalla et al. (2005; 2006), who extended a 2-party solution is to the 3-party case. Shortly after, Abdalla and Pointcheval (2006) introduced a group protocol. Further, Abdalla et al. (2007) proposed a generic compiler that enables the derivation of an authenticated group key establishment protocol from an arbitrary authenticated 2-party key establishment. This compiler is indeed inspired by the above Burmester-Desmedt rationale, but adds extra features and attains very strong security guarantees. In particular, from a password-authenticated 2-party key establishment the compiled protocol is a password-authenticated group key establishment, that has thus been derived without adding idealizing assumptions or high-entropy secrets for authentication. The construction suggested by Abdalla et al. (2007) builds on the use of non-interactive and non-malleable commitments, which in the CRS model are known to be implementable through IND-CCA2 secure encryption schemes. Nam et al. (2011) identified and addressed a security problem in the original formulation of this compiler.

Aiming at eluding idealized assumptions, we present here a provably secure password-authenticated protocol for group key establishment in the common reference string (CRS) model. The three-round protocol we propose considers a fully asynchronous network with an active adversary. We build upon a one-round construction by Katz and Vaikuntanathan (2013) that can (as proven by Ben Hamouda et al. (2013)) be implemented from Cramer-Shoup ciphertexts; focusing here on the theoretical design and security proof, we refer to these papers for a detailed discussion on how to actually derive a concrete implementation of our protocol. In a nutshell, our construction can be taken for a generalization of Katz and Vaikuntanathan's scheme to a group setting, compiled through the ideas of Abdalla et al. (2007) which in turn build on the Burmester-Desmedt rationale.

Related schemes. Several group key establishment protocols for the scenario considered here have been proposed (Bresson et al., 2002; Abdalla et al., 2006; Dutta and Barua, 2006). Many of these initial constructions are based on the random oracle or the ideal cipher model, while few constructions rely on standard assumptions (Abdalla et al., 2007; Abdalla and Pointcheval, 2006). Another interesting proposal is that of Gorantla et al. (2010), which also focuses on achieving a security proof without idealized assumptions, but assumes long-term high-entropy secrets are available for authentication. 


\section{Phase 1: Two-party key establishments.}

AKE: All indices are to be taken in a cycle, i.e., $U_{n+1}=U_{1}$, etc.

For $i=1, \ldots, n$, execute $\operatorname{AKE}\left(U_{i}, U_{i+1}\right)$ —as a result, each user $U_{i}$ holds two keys $\vec{K}_{i}, \overleftarrow{K}_{i}$ shared with $U_{i+1}$ respectively $U_{i-1}$.

\section{Phase 2: Group key establishment.}

Computation: Each $U_{i}$ computes

$$
X_{i}:=\vec{K}_{i} \oplus \overleftarrow{K}_{i}
$$

Broadcast: Each $U_{i}$ broadcasts $\left(U_{i}, X_{i}\right)$.

Computation: Each $U_{i}$ sets $K_{i}:=\overleftarrow{K}_{i}$ and computes $n-1$ values

$$
K_{i-j}:=\overleftarrow{K}_{i} \oplus X_{i-1} \oplus \cdots \oplus X_{i-j} \quad(j=1, \ldots, n-1)
$$

which defines a master key

$$
K:=\left(K_{1}, \ldots, K_{n}\right),
$$

and extracts a session key $s k_{i}$ from $K$.

Fig. 1. Burmester-Desmedt construction: the high-level idea.

Table 1 compares our construction with related previous ones 1 in terms of theoretical assumption, communication complexity (number of rounds) and authentication method.

1.3. Paper outline. In the subsequent section we recall the basic components of the considered security framework, addressing specifics of password-based authentication. Thereafter, in Section 3 we describe the basic tools needed for our construction: smooth projective hashing, labeled public-key encryption and non-malleable commitments. Finally, in Section 4 we present our password-authenticated constant-round protocol for group key establishment along with a security proof in the CRS model.

\section{Security model and security goals}

The theoretical model underlying our proof is basically adapted from Katz et al. $(2001 ; 2006)$, building in turn on Bellare and Rogaway (1994) or Bellare et al. (2000). We assume that a common reference string CRS is available that, similarly as in the work of Gennaro and Lindell (2003a), encodes

(i) the information needed for implementing a non-malleable commitment scheme,

(ii) a uniformly distributed random element from a family of universal hash functions, and

\footnotetext{
${ }^{1}$ We have excluded Dutta and Barua (2006), as security flaws on this scheme were found later by Abdalla et al. (2006).
}

(iii) two values $v_{0}, v_{1}$ that will serve as input for a pseudorandom function.

Also, a dictionary $\mathcal{D} \subseteq\{0,1\}^{*}$ is assumed to be publicly known. We model the dictionary $\mathcal{D}$ to be efficiently recognizable and of constant or polynomial size. In particular, we must assume that a polynomially bounded adversary is able to exhaust $\mathcal{D}$. The polynomial-sized set $\mathcal{U}=\left\{U_{1}, \ldots, U_{n}\right\}$ of users is assumed to share a common password $p w \in \mathcal{D}$. Further users, not contained in $\mathcal{U}$ and not knowing the shared password, can be simulated by the adversary. For the sake of simplicity, we adopt the common assumption that $p w$ has been chosen uniformly at random from $\mathcal{D}$, therewith slightly simplifying the formalism.

2.1. Communication model and adversarial capabilities. Users are modelled as probabilistic polynomial time (ppt) Turing machines Each user $U \in \mathcal{U}$ may execute a polynomial number of protocol instances in parallel. To refer to instance $s_{i}$ of a user $U_{i} \in \mathcal{U}$ we use the notation $\Pi_{i}^{s_{i}}(i \in \mathbb{N})$.

Protocol instances. A single instance $\Pi_{i}^{s_{i}}$ can be taken for a process executed by $U_{i}$. To each instance we assign seven variables:

used $_{i}^{s_{i}}$ indicates whether this instance is or has been used for a protocol run; the used ${ }_{i}^{s_{i}}$ flag can only be set through a protocol message received by the instance due to a call to the Send-oracle (see below);

\footnotetext{
${ }^{2}$ All our proofs hold for both uniform and non-uniform machines.
} 
Table 1. Comparison: our proposal vs other group key exchange protocols.

\begin{tabular}{|l|l|r|r|}
\hline \multicolumn{1}{|c|}{ Scheme } & \multicolumn{1}{|c|}{ Model } & \# Rounds & \multicolumn{1}{c|}{ Authentication } \\
\hline \hline Bressonn et al., 2002 & ROM & linear in \# of users & password \\
Abdalla et al., 2006 & ROM & 4 & password \\
Abdalla and Pointcheval, 2006 & Standard & 5 & password \\
Gorantla et al., 2010 & Standard & 2 & high-entropy secret \\
Proposed scheme & Standard & 3 & password \\
\hline
\end{tabular}

state $_{i}^{s_{i}}$ keeps the state information needed during the protocol execution;

$\operatorname{term}_{i}^{s_{i}}$ shows if the execution has terminated;

$\operatorname{sid}_{i}^{s_{i}}$ denotes a possibly public session identifier that can serve as identifier for the session key $\mathrm{sk}_{i}^{s_{i}}$;

$\operatorname{pid}_{i}^{s_{i}}$ stores the set of identities of those users that $\Pi_{i}^{s_{i}}$ aims at establishing a key with-including $U_{i}$ himself 3

$\operatorname{acc}_{i}^{s_{i}}$ indicates if the protocol instance was successful, i. e., the user accepted the session key;

$\mathrm{sk}_{i}^{s_{i}}$ stores the session key once it is accepted by $\Pi_{i}^{s_{i}}$. Before acceptance, it stores a distinguished NULL value.

For more details on the usage of the variables we refer to the work of Bellare et al. (2000).

Communication network. Arbitrary point-to-point (peer-to-peer) connections among the users are assumed to be available. Thus, the network topology is that of a complete graph. We assume the network to be non-private, however, and fully asynchronous. More specifically, it is controlled by the adversary, who may delay, insert and delete messages at will.

Adversarial capabilities. We restrict to ppt adversaries. The capabilities of an adversary $\mathcal{A}$ are made explicit through a number of oracles allowing $\mathcal{A}$ to communicate with protocol instances run by the users:

Send $\left(U_{i}, s_{i}, M\right)$ This sends message $M$ to the instance $\Pi_{i}^{s_{i}}$ and returns the reply generated by this instance. If $\mathcal{A}$ queries this oracle with an unused instance $\Pi_{i}^{s_{i}}$ and $M$ being the string "Start", the used ${ }_{i}^{s_{i}}$-flag is set, and the initial protocol message of $\Pi_{i}^{s_{i}}$ is returned.

Execute $\left(\left\{\Pi_{u_{1}}^{s_{u_{1}}}, \ldots, \Pi_{u_{\mu}}^{s_{u_{\mu}}}\right\}\right)$ This executes a complete protocol run among the specified unused instances of the respective users. The adversary obtains a

\footnotetext{
${ }^{3}$ Dealing with authentication through a shared password exclusively, we do not consider key establishments among strict subsets of $\mathcal{U}$. With $\operatorname{pid}_{i}^{s_{i}}:=\mathcal{U}$ being the only case of interest, in the sequel we do not make explicit use of $\operatorname{pid}_{i}^{s_{i}}$ when defining partnering, integrity, etc.
}

transcript of all messages sent over the network. A query to the Execute oracle is supposed to reflect a passive eavesdropping. In particular, no online-guess for the secret password can be implemented with this oracle.

Reveal $\left(U_{i}, s_{i}\right)$ yields the session key $\mathrm{sk}_{i}^{s_{i}}$.

Test $\left(U_{i}, s_{i}\right)$ Only one query of this form is allowed for an active adversary $\mathcal{A}$. Provided that $\mathrm{sk}_{i}^{s_{i}}$ is defined, (i. e., $\operatorname{acc}_{i}^{s_{i}}=$ true and $\mathrm{sk}_{i}^{s_{i}} \neq$ NULL), $\mathcal{A}$ can execute this oracle query at any time when being activated. Then with probability $1 / 2$ the session key $\mathrm{sk}_{i}^{s_{i}}$ and with probability $1 / 2$ a uniformly chosen random session key is returned.

2.2. Correctness, integrity and secrecy. Before we define correctness, integrity and secrecy, we introduce partnering to express which instances are associated in a common protocol session.

Partnering. We adopt the notion of partnering from Bohli et al. (2007). Namely, we refer to instances $\Pi_{i}^{s_{i}}$, $\Pi_{j}^{s_{j}}$ as being partnered if both $\operatorname{sid}_{i}^{s_{i}}=\operatorname{sid}_{j}^{s_{j}}$ and $\operatorname{acc}_{i}^{s_{i}}=$ $\mathrm{acc}_{j}^{s_{j}}=$ true.

To avoid trivial cases, we assume that an instance $\Pi_{i}^{s_{i}}$ always accepts the session key constructed at the end of the corresponding protocol run if no deviation from the protocol specification occurs. Moreover, all users in the same protocol session should come up with the same session key, and we capture this in the subsequent notion of correctness.

Correctness. We call a group key establishment protocol $\mathcal{P}$ correct, if in the presence of a passive adversary $\mathcal{A}$, i.e., $\mathcal{A}$ must not use the Send oracle, the following holds: for all $i, j$ with both $\operatorname{sid}_{i}^{s_{i}}=\operatorname{sid}_{j}^{s_{j}}$ and $\operatorname{acc}_{i}^{s_{i}}=\operatorname{acc}_{j}^{s_{j}}=$ true, we have $\mathrm{sk}_{i}^{s_{i}}=\mathrm{sk}_{j}^{s_{j}} \neq$ NULL.

Key integrity. While correctness takes only passive attacks into account, key integrity does not restrict the adversary's oracle access: a correct group key establishment protocol fulfills key integrity, if with overwhelming probability all instances of users that have accepted with the same session identifier $\operatorname{sid}_{j}^{s_{j}}$ hold 
identical session keys $\mathrm{sk}_{j}^{s_{j}}$. Next, for detailing the security definition, we will have to specify under which conditions a Test-query may be executed.

Freshness. A Test-query should only be allowed to those instances holding a key that are not for trivial reasons known to the adversary. To this aim, an instance $\Pi_{i}^{s_{i}}$ is called fresh if the adversary never queried $\operatorname{Reveal}\left(U_{j}, s_{j}\right)$ with $\Pi_{i}^{s_{i}}$ and $\Pi_{j}^{s_{j}}$ being partnered.

The idea here is that revealing a session key from an instance $\Pi_{i}^{s_{i}}$ trivially yields the session key of all instances partnered with $\Pi_{i}^{s_{i}}$, and hence this kind of "attack" will be excluded in the security definition.

Security/key secrecy. Because of the polynomial size of the dictionary $\mathcal{D}$, we cannot prevent an adversary from correctly guessing the shared secret $p w \in \mathcal{D}$ with non-negligible probability. Our goal is to restrict the adversary $\mathcal{A}$ to online verification of password guesses. For a secure group key establishment protocol, we have to impose a corresponding bound on the adversary's advantage: The advantage $\operatorname{Adv}_{\mathcal{A}}(\ell)$ of a ppt adversary $\mathcal{A}$ in attacking protocol $\mathcal{P}$ is a function in the security parameter $\ell$, defined as

$$
\operatorname{Adv}_{\mathcal{A}}:=|2 \cdot \operatorname{Succ}-1| .
$$

Here Succ is the probability that the adversary queries Test on a fresh instance $\Pi_{i}^{s_{i}}$ and guesses correctly the bit $b$ used by the Test oracle in a moment when $\Pi_{i}^{s_{i}}$ is still fresh.

Now, to capture key secrecy we follow the approach of Gennaro and Lindell (2003a). The intuition behind the definition is that the adversary must not be able to test (on-line) more than one password per protocol instance. This approach is stricter than the one taken by Abdalla et al. (2006) in the sense that we do not tolerate a constant number $>1$ of on-line guesses per protocol instance.

2.3. Strongly universal 2 projective hashing. Kurosawa and Desmedt introduced the notion of strongly universal 2 projective hash families, building on the previous work of Cramer and Shoup (2002) on different flavors of projective hashing. Projective hash families are usually understood as related to hard subset membership problems and in this fashion serve as a basis for several provably secure cryptographic constructions (Cramer and Shoup, 2002; Gennaro and Lindell, 2003a; González Vasco et al., 2005; Kalai, 2005; Kurosawa and Desmedt, 2004).

Definition 1. A subset membership problem $\mathcal{I}$ is a specification of a collection of probability distributions $\left\{I_{\ell}\right\}_{\ell \in \mathbb{N}}$, where for each $\ell, I_{\ell}$ is a probability distribution over instance descriptions. An instance description $\Lambda$ specifies:
1. Two finite, non-empty sets $X_{\ell}, L_{\ell} \subseteq\{0,1\}^{\operatorname{poly}(\ell)}$ with $L_{\ell} \subseteq X_{\ell}$.

2. Two probability distributions $D\left(L_{\ell}\right)$ and $D\left(X_{\ell} \backslash L_{\ell}\right)$ over $L_{\ell}$ and $X_{\ell} \backslash L_{\ell}$, respectively.

3. A set $W_{\ell} \subseteq\{0,1\}^{\text {poly }(\ell)}$, together with an NP-relation $R_{\ell} \subseteq X_{\ell} \times W_{\ell}$ such that $x \in L_{\ell}$ if and only if there exists $w \in W_{\ell}$ such that $(x, w) \in R_{\ell}$.

The above definition is taken from Gennaro and Lindell (2003a) and deviates slightly from that of Cramer and Shoup (2002). Again following Gennaro and Lindell (2003a), we will only be interested in subset membership problems that are efficiently samplable, that is, for which probabilistic polynomial-time algorithms for the following tasks are available:

1. Upon input $1^{\ell}$, sample an instance $\Lambda$ from $I_{\ell}$,

2. Upon input $1^{\ell}$ and an instance $\Lambda$, sample $x \in L_{\ell}$ according to $D\left(L_{\ell}\right)$, together with a witness $w \in W_{\ell}$ for $x$.

3. Upon input $1^{\ell}$ and an instance $\Lambda$, sample a value $x \in$ $X_{\ell} \backslash L_{\ell}$ according to $D\left(X_{\ell} \backslash L_{\ell}\right)$.

Our definition of a hard subset membership problem is identical to the one in the work of Gennaro and Lindell (2003a) and basically says that within $X_{\ell}$ distinguishing random elements inside and outside $L_{\ell}$ is hard.

Definition 2. Let $\mathcal{I}$ be a subset membership problem as above. Then we say that $\mathcal{I}$ is a hard subset membership problem, provided that the ensembles $\left\{\left(\Lambda_{\ell}, x_{\ell}\right)\right\}_{\ell \in \mathbb{N}}$ and $\left\{\left(\Lambda_{\ell}, \hat{x}_{\ell}\right)\right\}_{\ell \in \mathbb{N}}$ are computationally indistinguishable for $\Lambda_{\ell}, x_{\ell}$ and $\hat{x}_{\ell}$ sampled according to $I_{\ell}, D\left(L_{\ell}\right)$ and $D\left(X_{\ell} \backslash L_{\ell}\right)$ respectively.

Subsequently, we make use of subset membership problems, where the set $X_{\ell}$ comes along with a certain type of partition:

Definition 3. Let $\mathcal{I}$ be a subset membership problem as above and suppose that $X_{\ell}=C_{\ell} \times \mathcal{D}_{\ell}$. Further, for each $p w \in \mathcal{D}_{\ell}$ denote by $X_{\ell}(p w)$ (resp., $L_{\ell}(p w)$ ) the set of pairs $(c, p w) \in X_{\ell}$, (resp., $\left.(c, p w) \in L_{\ell}\right)$. The distributions induced by $D\left(L_{\ell}\right)$ and $D\left(X_{\ell} \backslash L_{\ell}\right)$ in $X_{\ell}(p w)$ and $L_{\ell}(p w)$ are denoted by $D\left(L_{\ell}(p w)\right)$ and $D\left(X_{\ell}(p w) \backslash L_{\ell}(p w)\right)$.

We say that $\mathcal{I}$ is a hard partitioned subset membership problem, provided that for every $p w \in \mathcal{D}_{\ell}$, the ensembles $\left\{\left(\Lambda_{\ell}, x_{\ell}\right)\right\}_{\ell \in \mathbb{N}}$ and $\left\{\left(\Lambda_{\ell}, \hat{x}_{\ell}\right)\right\}_{\ell \in \mathbb{N}}$ are computationally indistinguishable for $\Lambda_{\ell}, x_{\ell}$ and $\hat{x}_{\ell}$ being sampled according to $I_{\ell}, D\left(L_{\ell}(p w)\right)$ and $D\left(X_{\ell}(p w) \backslash\right.$ $\left.L_{\ell}(p w)\right)$, respectively.

This definition of hard partitioned subset membership problems is taken from Gennaro and 
Lindell (2003a) and captures the situation where each set $X_{\ell}$ can actually be partitioned into disjoint sets of hard problems. As Gennaro and Lindell (2003a) do, we stress here that the projective hash functions considered in the sequel will not take this partitioning into account. Moreover, in accordance with the results of Cramer and Shoup (2002) (and differing from those of Gennaro and Lindell (2003a)) we use a definition of projective hash families where the projection function $\alpha$ has only one argument.

Definition 4. Let $X, \Pi$ be finite non-empty sets and $K$ some finite index set. Consider a family $H=\left\{H_{k}\right.$ : $X \longrightarrow \Pi\}_{k \in K}$ of mappings from $X$ into $\Pi$, and let $\alpha: K \longrightarrow S$ be a map from $K$ into some finite non-empty set $S$ (which may be seen as a projection).

Then, given a subset $L \subseteq X$, we refer to the tuple $\mathbf{H}=(H, K, X, L, \Pi, S, \alpha)$, as projective hash family (PHF) for $(X, L)$ if for all $k \in K, x \in L$ the value $H_{k}(x)$ is determined by $\alpha(k)$.

We are mainly interested in a special type of projective hash families, which by Kurosawa and Desmedt (2004) are called strongly universal 2 .

Definition 5. Let $\mathbf{H}=(H, K, X, L, \Pi, S, \alpha)$ be a PHF. Then we refer to $\mathbf{H}$ as strongly universal ${ }_{2}$ if for $k \in K$ chosen uniformly at random, for any $x, x^{*} \in X \backslash L, x \neq$ $x^{*}$ the random variables

- $\xi_{k}:=H_{k}(x)$, conditioned on $\alpha(k)$,

- $\eta_{k}$, the variable $\xi_{k}$ conditioned on both $\alpha(k)$ and $H_{k}\left(x^{*}\right)$,

are statistically close to the uniform distribution over $\Pi$.

In the sequel, we will consider only projective hash families which are efficient in the sense of Gennaro and Lindell (2003a), i.e., there are efficient algorithms available for sampling uniformly at random elements from $K$, computing $\alpha$ and evaluating $H_{k}$ at a given $x \in X$ provided that

- either $k$ is given as an input, or

- $x \in L$ and $(x, w), \alpha(k)$ are given as input, where $w$ is a witness for $x$.

It is worth noting here that, in combination with a hard subset membership problem, the strongly universal ${ }_{2}$ property guarantees that, for $x \neq x^{*} \in X$ and $\alpha(k), H_{k}\left(x^{*}\right)$ the value $H_{k}(x)$ is indistinguishable from random unless a corresponding witness is known 4

\footnotetext{
${ }^{4}$ Smooth projective hashing would not suffice to guarantee independence with arbitrary different inputs $x \neq x^{*} \in X$
}

2.4. Strongly universal 2 hashing from nonmalleable commitments. Another essential component of Gennaro and Lindell's construction and of our proposal are non-interactive and non-malleable commitment schemes. Roughly speaking, they should fulfill the following requirements:

1. Every commitment $c$ defines at most one value (decommit $(c)$ ) (i.e., the scheme must be perfectly binding).

2. If an adversary receives several commitments to a value $\nu$, he must not be able to output a commitment to a value $\beta$ related to $\nu$ in a known way (that is, it must achieve non-malleability for multiple commitments).

In the common reference string model, the above commitment schemes can be constructed from any public-key encryption scheme that is non-malleable and secure for multiple encryptions (in particular, from any IND-CCA2 secure public-key encryption scheme).

We briefly recall Gennaro and Lindell's proposal for constructing smooth projective hash families, given a suitable commitment scheme as above: Let $\mathcal{C}$ be a commitment scheme fulfilling the conditions above (thus, we are in the common reference string model). Let $\mathcal{D}$ a fixed message (password) space. We denote by $C_{\rho}(p w ; r)$ a commitment to $p w \in \mathcal{D}$ using randomness $r$ and common reference string $\rho$. Let $C_{\rho}$, let us denote the set of all strings that may be output by $\mathcal{C}$ when the common reference string is $\rho$. For an efficiently recognizable superset $C_{\rho}^{\prime} \supseteq C_{\rho}$, define $X_{\rho}:=C_{\rho}^{\prime} \times \mathcal{D}$ and let

$$
L_{\rho}:=\left\{(c, p w) \in C_{\rho} \times \mathcal{D} \mid \exists r: c=C_{\rho}(p w ; r)\right\} \subseteq X_{\rho} .
$$

We consider a subset membership problem defined as follows. For each $\ell \in \mathbb{N}$ a common reference string $\rho$ (of polynomial size in $\ell$ ) is selected. Further, for each $p w \in \mathcal{D}$ define $D\left(X_{\rho}(p w) \backslash L_{\rho}(p w)\right)$ (resp., $D\left(L_{\rho}(p w)\right)$ ) as the distribution induced by choosing random $r$ and computing $\left(C_{\rho}\left(0^{|p w|} ; r\right), p w\right)$ (resp., $\left.\left.\left.C_{\rho}(p w ; r), p w\right)\right)\right)$. As argued by Gennaro and Lindell (2003a), it is easy to see that the hiding property of the commitment scheme yields the following.

Proposition 1. Let $\mathcal{C}$ be a non-interactive and nonmalleable perfectly binding commitment scheme. Consider the above subset membership problem $\mathcal{I}$, where for each $\rho$ the set $X_{\rho}$ is partitioned by the sets $\left\{C_{\rho}^{\prime} \times\right.$ $\{p w\}\}_{p w \in \mathcal{D}}$. Then $\mathcal{I}$ is a hard partitioned subset membership problem.

Now, assume we have a strongly universal ${ }_{2}$ projective hash family defined with respect to $\left(X_{\rho}, L_{\rho}\right)$ as follows: Let $K$ be the key space, and for every $k \in K$ define

$$
H_{k}: C_{\rho}^{\prime} \times \mathcal{D} \longrightarrow G,
$$


where $G$ is a finite Abelian group of superpolynomial size. For the security proof of our protocol we need an analog of the result of Gennaro and Lindell (2003a, Lemma 3.1). Namely, we need that given a projection $\alpha(k) \in S$ and two valid commitments $c_{1}$ and $c_{2}$ on the same password $p w$, the values $H_{k}\left(c_{1}, p w\right)$ and $H_{k}\left(c_{2}, p w\right)$ be computationally indistinguishable from random (independent) values, provided appropriate witnesses are not known. Note that if the commitments are invalid (and hence $\left(c_{1}, p w\right)$ and $\left(c_{2}, p w\right)$ are outside $L)$, this follows trivially from the definition of strongly universal ${ }_{2}$. For valid commitments, this is a consequence of the hard subset membership problem.

Lemma 1. Let $\mathcal{I}$ be the hard partitioned subset membership problem described above. With each instance $\Lambda=(X, D(X \backslash L), L, D(L), W, R)$, associate the above strongly universal ${ }_{2}$ projective hash family $\mathbf{H}=$ $(H, K, X, L, G, S, \alpha)$ for $(X, L)$. Let $M$ be a ppt oracle machine, and define the following experiments:

$\operatorname{Exp}-\operatorname{Hash}(M): \quad$ An instance $\Lambda=(X, D(X \backslash$ $L), L, D(L), W, R)$ is selected from $I_{\ell}$. Then $M$ is given access to three oracles $\Omega_{L}$, Project and Hash :

$\Omega_{L}:$ When queried with a value $p w \in \mathcal{D}$, it outputs $C_{\rho}(p w, r)$ with the pair $\left(C_{\rho}(p w, r), p w\right)$ being selected according to $D(L(p w))$ from $L(p w)$.

Project: Chooses a key $k \in K$ uniformly at random and returns $\alpha(k)$.

Hash: When queried with input $(p w, c, \alpha(k))$, it first checks if $c$ was output by $\Omega_{L}$ on input pw, and $\alpha(k)$ has been output by Project. If at least one of this is the case, Hash outputs $H_{k}(c, p w)$. Otherwise, Hash outputs nothing.

The output of the experiment is the output of $M$.

$\operatorname{Exp}-U n i f(M):$ Exactly as above, except that the Hash oracle is substituted by an oracle Unif which first checks whether the input $c$ was output by $\Omega_{L}$ on input pw, and that $\alpha(k)$ has been output by Project. If both are true, Unif outputs a uniformly distributed random $g \in G$; moreover, for two different calls with the same input $\alpha(k)$, the corresponding two random group elements will be selected independently. If only one of $c$ and $\alpha(k)$ was output by an oracle, Unif outputs $H_{k}(c, p w)$, otherwise, Unif outputs nothing.

Then, the above experiments are computationally indistinguishable, that is, for any ppt oracle machine $M$, for any value $v$ it may output,

$$
|\operatorname{Pr}[\operatorname{Exp}-U n i f(M)=v]-\operatorname{Pr}[\operatorname{Exp}-\operatorname{Hash}(M)=v]|
$$

is negligible in the security parameter $\ell$.
Proof. This proof is a straightforward variation of the proof by Gennaro and Lindell (2003a, Lemma 3.1). As they do, we define the experiments Exp-Unif ${ }_{X \backslash L}$ and Exp-Hash $_{X \backslash L}$ by replacing the oracle $\Omega_{L}$ by an oracle $\Omega_{X \backslash L}$ defined in the obvious way. Now, as we are dealing with a hard partitioned subset membership problem, both

$$
\begin{aligned}
\mid \operatorname{Pr}\left[\operatorname{Exp}_{-H_{X}} \operatorname{Hash}_{X \backslash L}(M)\right. & =v] \\
& -\operatorname{Pr}[\operatorname{Exp}-H \operatorname{Hash}(M)=v] \mid
\end{aligned}
$$

and

$$
\begin{aligned}
\mid \operatorname{Pr}\left[\operatorname{Exp} U_{X \backslash L}(M)=\right. & v] \\
& -\operatorname{Pr}[\operatorname{Exp}-U n i f(M)=v] \mid
\end{aligned}
$$

are negligible. Furthermore,

$$
\begin{aligned}
\mid \operatorname{Pr}\left[\operatorname{Exp}^{-H_{a s h} \backslash L}{ }_{X \backslash L}(M)\right. & =v] \\
& -\operatorname{Pr}\left[\operatorname{Exp}-U_{n i f}{ }_{X \backslash L}(M)=v\right] \mid
\end{aligned}
$$

is also negligible by the definition of strongly universal 2 , and putting it all together we have

$$
\begin{aligned}
& |\operatorname{Pr}[\operatorname{Exp}-U n i f(M)=v]-\operatorname{Pr}[\operatorname{Exp}-\operatorname{Hash}(M)=v]| \\
& \leq \mid \operatorname{Pr}[\operatorname{Exp}-\operatorname{Hash}(M)=v] \\
& -\operatorname{Pr}\left[\text { Exp-Hash }{ }_{X \backslash L}(M)=v\right] \mid \\
& +\mid \operatorname{Pr}\left[\operatorname{Exp}-\operatorname{Hash}_{X \backslash L}(M)=v\right] \\
& -\operatorname{Pr}\left[\operatorname{Exp}_{-U n i f}{ }_{X \backslash L}(M)=v\right] \mid \\
& +\mid \operatorname{Pr}\left[\operatorname{Exp}_{-U n i f}{ }_{X \backslash L}(M)=v\right] \\
& -\operatorname{Pr}[\operatorname{Exp}-\operatorname{Unif}(M)=v] \mid \text {, }
\end{aligned}
$$

from which the desired result follows.

\section{Smooth projective hashing and CCA-labeled encryption}

Smooth projective hash functions (SPHFs) were introduced by Cramer and Shoup (2002), and have resulted in a central tool for several provable secure constructions (Cramer and Shoup, 2002; Gennaro and Lindell, 2003a; González Vasco et al., 2005; Kalai, 2005; Kurosawa and Desmedt, 2004; Ben Hamouda et al., 2013; Blazy and Chevalier, 2015). Informally, consider a family of functions $\left\{H_{k}: X \longrightarrow \mathbb{G}\right\}_{k \in K}$ indexed by a countable set $K$, which acts on the elements of a set $X$, for a given group $\mathbb{G}$, both being finite. Now, given a distinguished language $L$ defined over $X$, the above family defines a SPHF for $(X, L)$ if there are four efficient algorithms, which in turn allow for selecting a hashing key $k$, computing a projection $\alpha(k)$ of it, and evaluating $H_{k}$ on any $x \in X$ either from the hashing key $k$ or from a tuple $(w, \alpha(k))$ where $w$ is a witness that evidences $x \in L$. 
As a correctness requirement, indeed whenever $w$ is a valid $L$-witness for $x$, the hashing values computed directly from $k$ and from $(w, \alpha(k))$ must coincide. However, the smoothness property implies that if $x$ is not in $L, H_{k}(x)$ must be statistically indistinguishable from an element selected uniformly at random in the range of $\mathbb{G}$ even knowing the projection key $\alpha(k)$. There have been different definitions for SPHF, depending essentially on:

- how the projection key $\alpha(k)$ is defined: adaptively (depending both on $k$ and $x$ ) or non adaptively (depending only on $k$ );

- whether $x$ may be computed adaptively from $\alpha(k)$.

In this work, we will stick to the definition of Katz and Vaikuntanathan (2013), for which $\alpha(k)$ will only depend on the hash key $k$ and yet the word $x$ may be chosen from $\alpha(k)$ in an adaptive way. In the sequel, we introduce the main notions needed for our construction following essentially (Katz and Vaikuntanathan, 2013), therefore not aiming at full generality. Most definitions in this section are verbatim taken from Katz and Vaikuntanathan (2013).

We start by stating what we mean by a labelled public-key encryption scheme, which fits applications in which both plaintexts and ciphertexts are tagged by labels in a consistent manner (Shoup, 2006). The different security notions for public-key encryption can easily be adapted for labeled schemes (see, e.g., Abdalla and Pointcheval, 2006). Informally, the main point for adapting security definitions is to modify the challenge construction phase assuming the adversary must provide not only two plain texts $m_{0}$ and $m_{1}$, but also a label $\mathrm{I}$. As a result, when considering CCA security, he will not be allowed to query his decryption oracle on any pair (label, $c$ ) where $c$ has been output by the encryption oracle on an input involving the label I.

Definition 6. A labeled public-key encryption scheme is a tuple of probabilistic polynomial time algorithms (Gen, Enc, Dec) such that

- Gen, the key-generation algorithm, takes as input a security parameter $1^{n}$ and returns a pair of (public, secret) keys $(p k, s k)$. This is denoted by $(p k, s k) \leftarrow$ $\operatorname{Gen}\left(1^{n}\right)$.

- Enc, the encryption algorithm, takes as input a public key $p k$, a label label and a plaintext $m$ and returns a ciphertext $C$. We write: $C \leftarrow \operatorname{Enc}(p k$, label, $m)$, or $C \leftarrow \operatorname{Enc}(p k$, label, $m, r)$, if we want to make explicit the randomness $r$ possibly involved in the computation.

- Dec, the decryption algorithm, takes as input a secret key $s k$, a label label and a ciphertext $C$, and returns a plaintext $m$ or an error message $\perp$. In symbols; $m \leftarrow \operatorname{Dec}(s k$, label, $C)$.

Moreover, we assume that for all $(p k, s k)$ output by Gen $\left(1^{n}\right)$, any label, any plaintext $m$, and any $C$ output by $\operatorname{Enc}(p k$, label, $m)$, there holds $\operatorname{Dec}(s k$, label, $C)=m$. At this, plain texts, cipher texts, and labels are assumed to be bitstrings of length polynomial in $n$. The plain text and label spaces are supposed to be implicitly defined (and may depend on the public key).

Building on a CCA-secure labeled encryption scheme (as Katz and Vaikuntanathan, 2013), we define a hard subset-membership problem that will serve as a basis for the SPHF which is the main tool of our construction. We refer to Gennaro and Lindell (2003b) for the general definition of hard subset-membership problems.

Let $\mathcal{D} \subseteq\{0,1\}^{*}$ be a fixed dictionary and (Gen, Enc, Dec) a CCA-secure labeled encryption scheme. For any given public key output by Gen, $p k$, we denote by $C_{p k}$ the set of valid pairs (label, $C$ ) with respect to the public key $p k$ (thus, $C$ is a cipher text), and assume this set to be efficiently recognizable. Now, consider:

- $X=\{($ label, $C, p w) \mid$ (label, $C) \in C_{p k}$ and $p w \in$ D)

- $L_{p w}=\{($ label, Enc $(p k$, label, $p w), p w)$ where label $\in$ $\left.\{0,1\}^{*}\right\}$.

Note that $L=\bigcup_{p w \in \mathcal{D}} L_{p w} \subseteq X$. As the encryption scheme is CCA, it can be proven that $(X, L)$ define a hard subset-membership problem: for any probabilistic polynomial-time $\mathcal{A}$, he can only win with negligible probability the following experiment:

- Phase 1: Setup. $(p k, s k) \leftarrow \operatorname{Gen}\left(1^{n}\right)$ and $b \leftarrow\{0,1\}$ is selected uniformly at random. The adversary is given the public key and is also granted access to a $b$-encryption oracle, which, on any input (label, $m_{0}, m_{1}$ ), with $m_{0}$ and $m_{1}$ of the same bit size, will output $\operatorname{Enc}\left(p k\right.$, label, $\left.m_{b}\right)$.

- Phase 2: Challenge. $\mathcal{A}$ selects two passwords $p w_{0}$ and $p w_{1}$ from $\mathcal{D}$ (assumed to be of the same bit size), and a label label. He is presented with an encryption $C \leftarrow \operatorname{Enc}\left(p k\right.$, label, $\left.p w_{b}\right)$.

- Phase 3: Output. On top of the $b$-encryption oracle, $\mathcal{A}$ has now access to a decryption oracle holding the secret key $s k$, which he cannot query with the input (label, $C$ ). He outputs a guess $b^{\prime}$ for the bit $b$.

Now, the above ingredients will be used to define a $\mathrm{KV}-\mathrm{SPHF}$ for any given public key $p k$. 
Definition 7. Let $X$ be a non-empty set, $\mathbb{G}$ be a group and $K$ some index set (all finite). Consider a family $H=$ $\left\{H_{k}: X \longrightarrow \mathbb{G}\right\}_{k \in K}$ of mappings from $X$ into $\mathbb{G}$, and let $\alpha: K \longrightarrow S$ be a map from $K$ into some finite non-empty set $S$ (which may be seen as a projection). Given a subset $L \subseteq X$, we refer to the tuple $\mathbf{H}=(H, K, X, L, G, S, \alpha)$, as smooth projective hash family (SPHF) for $(X, L)$ if there are efficient algorithms

- HashKG: selects uniformly at random a hash key $k \in$ $K$

- ProjKG $(k)$ : computes a projection key $\alpha(k)$,

- Hash $(k, x)$ : outputs $H_{k}(x)$ computed from the hashing key $k$.

- ProjHash $(\alpha(k), x, w)$ : outputs $H_{k}(x)$ from the projection key $\alpha(k)$, provided that $w$ is a valid witness evidencing $x \in L$.

Moreover, for any function $f: S \longrightarrow X \backslash$ $L$, the following distributions have statistical difference negligible in the security parameter $n$ :

$$
\left\{k \leftarrow \text { HashKG }, s \leftarrow \operatorname{ProjKG}(k):\left(s, H_{k}(f(s))\right)\right\}
$$

and

$$
\{k \leftarrow \operatorname{HashKG}, s \leftarrow \operatorname{ProjKG}(k), g \leftarrow G:(s, g)\} .
$$

Remark 1. As neatly explained by Ben Hamouda et al. (2013), the main difference between the above definition of smoothness and previous ones that can be found in the literature is that

- KV-SPHF (Katz and Vaikuntanathan, 2013): the projection key does not depend on the word $C$ and furthermore the smoothness condition holds even if $C$ is constructed knowing $\alpha(k)$,

- CS-SPHF(Cramer and Shoup, 2002): the projection key $\alpha(k)$ does not depend on $C$, but $C$ must not depend on $\alpha(k)$,

- GL-SPHF(Gennaro and Lindell, 2003b): $\alpha(k)$ may depend on $C$.

In the work of Ben Hamouda et al. (2013), a new KV-SPHF is constructed from the labelled Cramer-Shoup encryption. Recall that KV-SPHFs were designed with the goal of achieving one-round PAKE. In order to do with just one round, the cipher text and the projection key for verifying the correctness of the partner's cipher text should be sent together, and thus be independent. Moreover, the smoothness property must hold in a scenario where the adversary can wait until it receives the partner's projection key before generating the cipher text.
Let us go back to the concrete instance of a hard subset membership problem as explicited above. At this, note that ProjHash $(\alpha(k)$, label, $C, p w, r)$ will output $H_{k}$ (label, C, pw) if and only if $r$ is a valid witness of (label, $C, p w)$, namely, if and only if $C \leftarrow \operatorname{Enc}(p k$, label, $p w, r)$. We will make use of the following technical lemma taken from Katz and Vaikuntanathan (2013), which in turn is a refinement of Lemma 3.4 of Gennaro and Lindell (2006). It roughly states that seeing many projection keys will not help in distinguishing (properly constructed) hashes from elements selected at random from $\mathbb{G}$, if appropriate witnesses are not known.

Lemma 2. Let LENC = (Gen, Enc, Dec) be a CCAlabeled public-key encryption scheme, $\rho=\rho(n)$ be a fixed polynomial function and $\mathcal{A}$ a probabilistic polynomial time adversary. For $b \in\{0,1\}$, we define the experiment $\operatorname{Exp}_{b}$ as

- Phase 1: Setup. Execute $(p k, s k) \leftarrow$ $\operatorname{Gen}\left(1^{n}\right)$, fix $\mathbf{H}=(H, K, X, L, \Pi, S, \alpha)$ a smooth projective hash function for $p k$ as above and forward this public key to $\mathcal{A}$.

- Phase 2: Challenge. Execute HashKG $\rho$ times, retrieving as output $k_{1}, \ldots, k_{\rho}$ which are in turn fed to $\operatorname{ProjKG}(\cdot)$. Feed the corresponding outputs $s_{1}, \ldots, s_{\rho}$ to $\mathcal{A}$.

- Phase 3: Output. During this phase, $\mathcal{A}$ is granted access to two oracles:

- $\mathcal{O}_{\text {Enc: }}$ a modified encryption oracle, which on input (label, pw) for any $p w \in \mathcal{D}$ outputs

* If $b=0: H_{k_{i}}$ (label, $\left.C, p w\right)$, for $i=$ $1, \ldots, \rho$, where $C \leftarrow \operatorname{Enc}(p k$, label, $p w)$,

* otherwise, if $b=1$ : $\rho$ values selected uniformly at random from $\mathbb{G}$.

- $\mathcal{O}_{\text {Dec }}$ : a CCA-decryption oracle for LENC, namely, this oracle may not be queried with any pair (label, $C$ ) where $C$ was obtained from the encryption oracle on query label, pw.

At the end of this phase, $\mathcal{A}$ outputs her guess $b^{\prime}$.

Then $\left|2 \operatorname{Pr}\left[b=b^{\prime}\right]-1\right|$ is negligible in the security parameter.

The previous lemma thus states that distinguishing between the two experiments $\operatorname{Exp}_{0}$ and $\operatorname{Exp}_{1}$ defined above is hard; thus hashes and random group elements are hard to distinguish even when having access to many projections. 


\section{Group key establishment protocol}

The protocol we propose builds on a CCA-labelled encryption scheme and a KV-SPHF $H=\left\{H_{k}\right\}_{k \in K}$ as described in the previous section. In particular, we assume the image of the hash functions $H_{k}$ to be contained in a finite Abelian group $\mathbb{G}$. Furthermore, we will use a family of universal hash functions $\mathcal{U H}$ that maps elements from $G^{n}$ onto a superpolynomial-sized set $\{0,1\}^{L}$, and a family of universal hash functions $\mathcal{U} \mathcal{H}^{\prime}$ that map elements from $\mathbb{G}$ onto a superpolynomial sized set $T$ of cardinality $|T| \leq \sqrt{|\mathbb{G}|}$. Similarly as Bresson et al. (2002), we impose an additional restriction on $\mathcal{U H}^{\prime}$, saying that there are no "bad indices" into the family $\mathcal{U} \mathcal{H}^{\prime}$. Namely, for each $\mathrm{UH}^{\prime} \in \mathcal{U} \mathcal{H}^{\prime}$ we require the following to hold: any ppt algorithm having $\mathrm{UH}^{\prime}$ as input has no more than a negligible probability to predict $\mathrm{UH}^{\prime}(g)$ for an (unknown) uniformly at random chosen $g \in \mathbb{G}$.

Example 1. Let $\mathbb{G}:=\mathbb{Z} / p \mathbb{Z}$ be the additive group of integers modulo an $\ell$-bit prime $p$, and let $L^{\prime}:=\lfloor\ell / 2\rfloor$. Choosing $T:=\{0,1\}^{L^{\prime}}$ to be the set of bitstrings of length $L^{\prime}$, the following family $\mathcal{U} \mathcal{H}^{\prime}$ considered by Carter and Wegman (1977) contains no "bad indices":

$$
\begin{aligned}
\mathcal{U} \mathcal{H}^{\prime}:=\left\{g \mapsto[a \cdot g+b]_{0 \rightarrow L^{\prime}-1}:\right. & \\
& a, b \in \mathbb{Z} / p \mathbb{Z} \text { with } a \neq 0\},
\end{aligned}
$$

where $[\cdot]_{0 \rightarrow L^{\prime}-1}$ denotes selection of $L^{\prime}$ least significant bits ("mod $2^{\left.L^{\prime} "\right)}$ ). The universality of $\mathcal{U H}^{\prime}$ is well known; cf., e.g., Carter and Wegman (1977). Moreover, as the case $a=0$ is excluded in the affine maps considered, for a uniformly distributed random $g \in \mathbb{G}$, also $a \cdot g+$ $b$ is uniformly distributed in $\mathbb{G}$, and the probability of predicting the correct value $\mathrm{UH}_{a, b}^{\prime}(g)=[a \cdot g+b]_{0 \rightarrow L^{\prime}-1}$ is negligible.

The CRS selects one universal hash function $\mathrm{UH}$ from the family $\mathcal{U H}$.

A collision-resistant pseudorandom function family. We use UH to select an index within a collision-resistant pseudorandom function family $\mathcal{F}=\left\{F^{\ell}\right\}_{\ell \in \mathbb{N}}$ as used by Katz and Shin (2005). We assume $F^{\ell}=\left\{F_{\eta}^{\ell}\right\}_{\eta \in\{0,1\}^{L}}$ to be indexed by $\{0,1\}^{L}$ and denote by $v_{0}=v_{0}(\ell)$ a publicly known value such that no ppt adversary can find two different indices $\lambda \neq \lambda^{\prime} \in\{0,1\}^{L}$ with $F_{\lambda}\left(v_{0}\right)=$ $F_{\lambda^{\prime}}\left(v_{0}\right)$ (see the work of Katz and Shin (2005) for more details). As Katz and Shin (2005) we use another public value $v_{1}$ (which, like $v_{0}$, can be included in the CRS) for deriving the session key. The family $\mathcal{U H}^{\prime}$ is used for confirming the auxiliary two-party keys $Z_{i, i-1}$ in Round 2 of our protocol without jeopardizing the password $p w$.

Commitments. Round 2 of our protocol uses another essential component already present in Gennaro and
Lindell's construction: non-interactive and non-malleable commitment schemes. Roughly speaking, they should fulfill the following requirements:

1. Every commitment $c$ defines at most one value (decommit $(c)$ ) (i.e., the scheme must be perfectly binding).

2. If an adversary receives several commitments to a value $\nu$, he must not be able to output a commitment to a value $\beta$ related to $\nu$ in a known way (that is, it must achieve non-malleability for multiple commitments).

In the common reference string model, the above commitment schemes can be constructed from any public-key encryption scheme that is non-malleable and secure for multiple encryptions (in particular, from any IND-CCA2 secure public-key encryption scheme) (see, for instance, the work of Gennaro and Lindell (2003b)) 5

Now we are ready to introduce our proposed construction. Our protocol is symmetric in the sense that all users perform the same steps. Figures 24 show the three rounds of our protocol. For the sake of readability, we do not explicitly refer to instances $s_{i}$ of users.

\section{Design comments}

The basic design of the protocol follows the construction by Burmester and Desmedt (1995), where the Diffie-Hellman key exchanges are replaced by the Katz-Vaikuntanathan one-round protocol (Katz and Vaikuntanathan, 2013) key exchange. A basic trick of our design is the construction of the master key as

$$
\mathrm{mk}=\left(Z_{1,2}, Z_{2,3}, \ldots, Z_{n-1, n}, Z_{n, 1}\right) .
$$

The original construction $\mathrm{mk}=\prod_{i=1, \ldots, n} Z_{i, i+1}$ can be determined by two malicious users as pointed out by Bohli et al. (2007). Thus, if an adversary guesses the password, he would be able to provoke pathological behaviors such that each protocol run ends up with exactly the same $\mathrm{mk}$ (and thus, identical $\mathrm{sid}_{i}, \mathrm{sk}_{i}$ ). Note that with the construction of $\mathrm{mk}$ proposed above, both $\mathrm{sid}_{i}$ and $\mathrm{sk}_{i}$ will be indistinguishable from random if a sole honest user is involved in the protocol run.

Let us further comment a bit about Round 2. At this stage, the idea is to broadcast commitments to the quotients $X_{i}$. This is to prevent an online attack on the protocol consisting only of Round 1 and Round 3, that allows to test two passwords using only one instance $\Pi_{i}^{s_{i}}$.

Further, note that the test ${ }_{i}$-values in Round 2 address attacks where one party did not receive the

\footnotetext{
${ }^{5}$ Actually, the encrypted values $c_{i, i+1}, c_{i, i-1}$ from Round 1 could as well have been defined as commitments constructed from such a commitment scheme. We preferred the encryption formulation to keep our formulation closer to that of Katz and Vaikuntanathan (2013).
} 


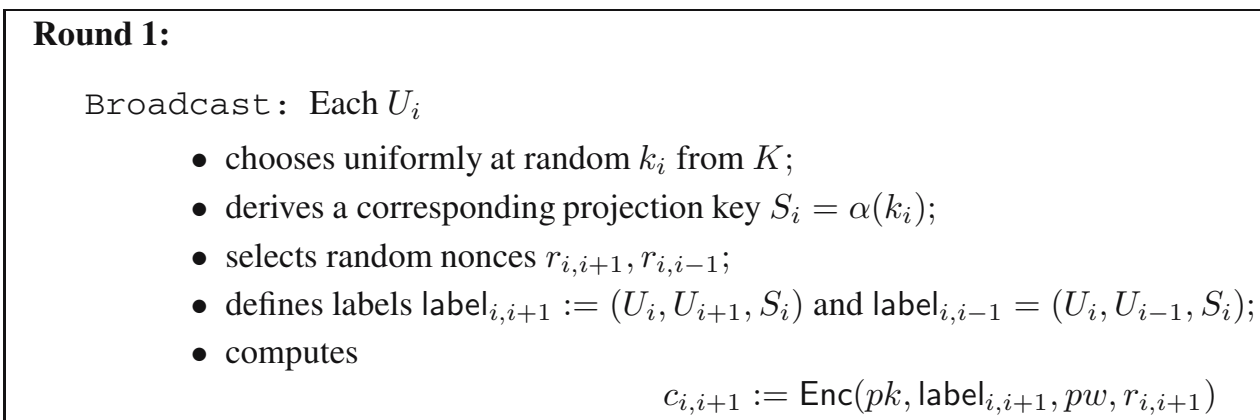

and

$$
c_{i, i-1}:=\operatorname{Enc}\left(p k, \text { label }_{i, i-1}, p w, r_{i, i-1}\right) ;
$$

- broadcasts $M_{i}^{1}:=\left(U_{i}, S_{i}, c_{i, i+1}, c_{i, i-1}\right)$.

Check: Each $U_{i}$

- waits until messages $M_{j}^{1}$ for all $U_{j}$ arrived;

- checks if the values $c_{i+1, i}$ and $c_{i-1, i}$ are valid encryptions

- If any of the checks fails, set $\operatorname{acc}_{i}:=$ false and terminate.

${ }^{a}$ At this, valid means a valid encryption of $p w$ with the expected $p k$ and label.

Fig. 2. Round 1: a password-authenticated 3-round protocol for group key establishment.

correct projection but rather a bogus one, inserted by the adversary. Note that this is needed despite assumptions on the projective hash function, for we have no guarantees if projections are not constructed from randomly selected elements $k \in K$ (for details, see Games 4 and 5 in the proof of Theorem (1). Finally, the random value $X_{i, 1}$ is needed if the check of a test $_{i}$-value fails. In this case, the true $X_{i, 0}$ must not be revealed. On the other hand, an adversary should not recognize if the check fails, as this would give a hint if the respective hash values and therefore a password that the adversary may have used was correct. This is again, to prevent that two passwords may be tested with one instance running the protocol.

\subsection{Security analysis.}

Theorem 1. With the prerequisites as described above, the protocol depicted in Figs. 2,4 is correct and achieves key secrecy and key integrity.

Proof. It is easy to see that the above protocol fulfills correctness and integrity, and the main part of our proof is devoted to key secrecy.

Correctness and integrity. Owing to the collision-resistance of the family $\mathcal{F}$, all oracles that accept with identical session identifier use with overwhelming probability the same index value $\mathrm{UH}(\mathrm{mk})$ and therewith also derive the same session key.

Key secrecy. We imagine a simulator that simulates the oracles and instances for the adversary. The proof is set up in terms of several experiments or games, where from game to game the simulator's behaviour somehow deviates from the previous. Following the standard notation, we denote by $\operatorname{Adv}\left(\mathcal{A}, G_{i}\right)$ the advantage of the adversary when confronted with Game $i$. The security parameter is denoted by $\ell$. Furthermore, we will index the Send oracle, denoting by Send $_{0}$ the Send query that initializes a protocol run and by Send $i$ a Send query that delivers a message of round $i$ for $i \in\{1,2,3\}$. As we must consider the session identifiers known to the adversary, we assume them to be part of the output of the final Send ${ }_{3}$ query.

For the sake of readability, we start by sketching an informal proof roadmap here:

- Game 0 is, as usual, modelling the real experiment faced by the adversary.

- Game 1 to Game 3 deal with the case of passive adversaries; thus, they progressively modify the Execute oracle: in Game 1 the two-party keys $Z_{i, j}$ are replaced by random bit strings, then in Game 2 the "real" password is substituted by another one and finally the session key is chosen in Game 3 uniformly at random. The adversary is unable to notice these steps, due to the hiding property of the commitment scheme, the semantic security of the encryption scheme and the fact that the values $Z_{i, j}$ and $X_{i}$ look anyway random to him.

Games 4 to 8 deal with adversaries that modify messages in Round 1. For all possible modifications, the 


\section{Round 2:}

Computation: Each $U_{i}$

- sets

$$
\text { label }_{i+1, i}:=\left(U_{i+1}, U_{i}, S_{i+1}\right)
$$

and

$$
\text { label }_{i-1, i}:=\left(U_{i-1}, U_{i}, S_{i-1}\right) \text {; }
$$

- derives two party keys:

$$
\begin{aligned}
& Z_{i, i+1}:=H_{k_{i}}\left(\operatorname{label}_{i+1, i}, p w, c_{i+1, i}\right) \cdot H_{k_{i+1}}\left(\text { label }_{i, i+1}, p w, c_{i, i+1}\right), \\
& Z_{i, i-1}:=H_{k_{i-1}}\left(\operatorname{label}_{i, i-1}, p w, c_{i, i-1}\right) \cdot H_{k_{i}}\left(\text { label }_{i-1, i}, p w, c_{i-1, i}\right) ;
\end{aligned}
$$

- sets $X_{i, 0}:=Z_{i, i+1} \cdot Z_{i, i-1}^{-1}$;

- chooses a random $X_{i, 1} \in G$;

- chooses random values $r_{i, 0}^{\prime}, r_{i, 1}^{\prime}$;

- computes commitments $C_{\rho}\left(U_{i}, X_{i, 0} ; r_{i, 0}^{\prime}\right)$ and $C_{\rho}\left(U_{i}, X_{i, 1} ; r_{i, 1}^{\prime}\right)$;

- chooses at random $\mathrm{UH}_{i}^{\prime} \in \mathcal{U H}^{\prime}$ and

- computes a test value test ${ }_{i}:=\mathrm{UH}_{i}^{\prime}\left(Z_{i, i-1}\right)$.

Broadcast: Each user $U_{i}$ broadcasts for a random bit $b$

$$
M_{i}^{2}:=\left(U_{i}, C_{\rho}\left(U_{i}, X_{i, b} ; r_{i, b}^{\prime}\right), C_{\rho}\left(U_{i}, X_{i, 1-b} ; r_{i, 1-b}^{\prime}\right), \text { test }_{i}, \mathrm{UH}_{i}^{\prime}\right) .
$$

Check: Each user $U_{i}$

- waits until messages $M_{j}^{2}$ for all $j$ arrived

- checks if

$$
\mathrm{UH}_{i+1}^{\prime}\left(Z_{i, i+1}\right)=\text { test }_{i+1} ;
$$

- if the check succeeds, set $\left(X_{i}, r_{i}^{\prime}\right):=\left(X_{i, 0}, r_{i, 0}\right)$, otherwise $\left(X_{i}, r_{i}^{\prime}\right):=\left(X_{i, 1}, r_{i, 1}\right)$.

Fig. 3. Round 2: a password-authenticated 3-round protocol for group key establishment.

recipient of the bogus message will randomize its value $X_{i}$, or the game is aborted. Also for correct messages, the values $Z_{i, i-1}$ and $Z_{i, i+1}$ are randomized. Table 2 gives an overview which game deals with which modifications caused by the adversary $\mathcal{A}$.

- Game 4 and 5 are concerned with the situation in which the adversary may insert projections in the first round. A malicious insertion of $S_{i-1}$ results in $U_{i}$ choosing $Z_{i, i-1}$ uniformly at random; in Game 5, if $U_{i}$ gets an adversarially sent $S_{i+1}$ the corresponding $X_{i}$ is chosen uniformly at random.

The adversary will not notice these changes in the simulation. In Game 4 the argument follows because inserting a projection will not help him distinguishing the $Z_{i, j}$ from values selected independently and uniformly at random, and thus messages from Round 2 will not help him detect the change. Furthermore, in both games the messages from Round 3 will not help the adversary in distinguishing. The adversary cannot prevent that with overwhelming probability the check in Round 2 will fail and thus in Round 3 uniform random values $X_{i, 1}$ will be broadcast.

- Game 6. Once ruled out the possibility of inserted projections, the simulator will now generate the two-party keys $Z_{i, j}$ independently and uniformly at random if the encryptions in round one were oracle-generated, i.e., honestly transmitted or replayed from other instances. Distinction between this game and Game 5 reduces to distinguishing between $\operatorname{Exp}_{0}$ and $\operatorname{Exp}_{1}$ from Lemma2

In the sequel, we will speak of "valid encryptions" to refer to encryptions of the correct $p w$ with the public key and label as expected.

- Game 7 deals with the case in which the adversary may insert an invalid encryption in Round 1. The simulator, detecting an invalid encryption, will choose $X_{i, 0}$ at random. This modification is due to the smoothness property not detectable by the 


\section{Round 3:}

Broadcast: Each user $U_{i}$ broadcasts $M_{i}^{3}:=\left(U_{i}, X_{i}, r_{i}^{\prime}\right)$.

Check: Each $U_{i}$

- checks that $X_{1} \cdots X_{n}=1$;

- checks the correctness of the commitments $C_{\rho}\left(U_{j}, X_{j} ; r_{j}^{\prime}\right)$;

- if at least one of theses checks fails, set $\operatorname{acc}_{i}:=$ false and terminate.

Computation: Each $U_{i}$ computes the values

$$
\begin{aligned}
Z_{i-1, i-2} & :=Z_{i, i-1} / X_{i-1}, \\
Z_{i-2, i-3} & :=Z_{i-1, i-2} / X_{i-2}, \\
& \vdots \\
Z_{i, i+1} & :=Z_{i+1, i+2} / X_{i+1},
\end{aligned}
$$

a master key

$$
\mathrm{mk}:=\left(Z_{1,2}, Z_{2,3}, \ldots, Z_{n-1, n}, Z_{n, 1}\right),
$$

and sets $\mathrm{sk}_{i}:=F_{\mathrm{UH}(\mathrm{mk})}\left(v_{1}\right), \operatorname{sid}_{i}:=F_{\mathrm{UH}(\mathrm{mk})}\left(v_{0}\right)$ and acc $i=$ true.

Fig. 4. Round 3: a password-authenticated 3-round protocol for group key establishment.

Table 2. Handling modified Round 1 messages in the proof of Theorem 1

\begin{tabular}{|c||c|c|c|c|}
\hline Game & $S_{i-1}$ & $S_{i+1}$ & $c_{i-1}$ & $c_{i+1}$ \\
\hline \hline 4 & replaced & $*$ & oracle-generated & oracle-generated \\
\hline 5 & $*$ & replaced & oracle-generated & oracle-generated \\
\hline 6 & $\checkmark$ & $\checkmark$ & oracle-generated & oracle-generated \\
\hline 7 & $*$ & $*$ & invalid & $*$ \\
& $*$ & $*$ & $*$ & invalid \\
\hline 8 & $*$ & $*$ & valid from $\mathcal{A}$ & valid from $\mathcal{A}$ \\
\hline
\end{tabular}

adversary from the messages exchanged.

- Game 8 deals with the case of valid encryptions $c_{i}$ generated by the adversary when he wins. This corresponds to a correct guess for the password.

- Game 9 aborts in case any encryption, commitment, projection or $X_{i}$-value is inserted by the adversary. The advantage of the adversary can only vary negligibly, as due to the non-malleability of the commitment scheme and the condition $X_{1} \cdots X_{n}=1$, the protocol would anyway abort with overwhelming probability.

- Game 10 and 11 argue similarly as in the passive case, once all malicious Send-queries are ruled out. First, in Game 10, encryptions from Round 1 are constructed using a randomly selected password. To conclude, the key generation is modified in Game 11 in that the session key is chosen uniformly at random. The adversary can only win by having inserted a valid commitment he constructed; otherwise he will not be able to tell the difference, given that $\mathrm{UH}$ is a universal hash function and (at least) one of its inputs $Z_{i, k}$ is a random group element. This concludes the proof.

Having outlined the structure of the proof, we are left to fill in the details:

Game 0. All oracles are simulated as defined in the model. Thus, $\operatorname{Adv}\left(\mathcal{A}, G_{0}\right)$ is exactly $\operatorname{Adv}(\mathcal{A})$.

Game 1. In this game, the simulation of the Execute oracle is modified. Instead of computing the values $Z_{i, i-1}, Z_{i, i+1}$ for $i=1, \ldots, n$ as specified in the protocol, they are chosen uniformly at random from $\mathbb{G}$. As a result, also the values $X_{i}$ will be random though fulfill the property $X_{1} \cdots X_{n}=1$ and the master key mk will be a randomly selected element from $\mathbb{G}^{n}$.

Let us now reason that the probability an adversary has of distinguishing between the values $X_{i}$ generated in Game 0 and the ones generated in Game 1 is no greater than the probability he has of distinguishing the experiments $\operatorname{Exp}_{0}$ and $\operatorname{Exp}_{1}$ from Lemma 2 Indeed, 
for a fixed common reference string and password the adversary cannot distinguish between $\operatorname{Exp}_{0}$ and $\operatorname{Exp}_{1}$, for $i=1, \ldots, n$. This means, seeing values $c_{i, i-1}, c_{i, i+1}$ and the projection $\alpha\left(k_{i}\right)$, he cannot tell $H_{k_{i}}\left(\right.$ label $\left._{\mathrm{i}+1, \mathrm{i}}, \mathrm{pw}, \mathrm{c}_{\mathrm{i}, \mathrm{i}-1}\right)$ and $H_{k_{i}}\left(\right.$ label $\left._{\mathrm{i}-1, \mathrm{i}}, \mathrm{pw}, \mathrm{c}_{\mathrm{i}-1, \mathrm{i}}\right)$ apart from independent random values; thus, the same applies to each element $X_{i}$ generated in Game 0.

Therefore, having a negligible probability of distinguishing between the two experiments we have

$$
\left|\operatorname{Adv}\left(\mathcal{A}, G_{1}\right)-\operatorname{Adv}\left(\mathcal{A}, G_{0}\right)\right| \leq \operatorname{negl}(\ell) .
$$

Game 2. At this juncture, the Execute oracle is again modified, so that a password $\widehat{p w}$ is chosen uniformly at random from $\mathcal{D}$. Further, each $c_{i, i+1}$ and $c_{i, i-1}$ are computed consistently. Due to the semantic security of the encryption scheme ENC, we again have

$$
\left|\operatorname{Adv}\left(\mathcal{A}, G_{2}\right)-\operatorname{Adv}\left(\mathcal{A}, G_{1}\right)\right| \leq \operatorname{negl}(\ell)
$$

Game 3. Let us consider a further modification of the Execute oracle. Namely, the simulator will assign to the instances a session key $\mathrm{sk}_{i}^{s_{i}} \in\{0,1\}^{\ell}$, chosen uniformly at random.

Note that even knowing all values $X_{i}$, still the value of at least one of the two-party keys $Z_{k, j}$ is indistinguishable from a random group element. Thus, with the leftover hash lemma, we see that the master key $\mathrm{mk}=\left(Z_{1,2}, \ldots, Z_{n, 1}\right)$ has sufficient entropy so that the output of the pseudorandom function $F_{\mathrm{UH}(\mathrm{mk})}$ is distinguishable from a random $\mathrm{sk}_{i}^{s_{i}}$ with negligible probability only,

$$
\left|\operatorname{Adv}\left(\mathcal{A}, G_{3}\right)-\operatorname{Adv}\left(\mathcal{A}, G_{2}\right)\right| \leq \operatorname{negl}(\ell) .
$$

By now the Execute oracle returns only random values, independent of the password, and instances used by an Execute-query hold only random session keys. The following games will deal with the Send oracle.

In the following we will call an encryption that was generated by the simulator oracle-generated and in accordance an encryption that was generated by the adversary adversary-generated. This can be checked efficiently by keeping a list of all encryptions the simulator generates. Furthermore, we call the encryption valid if it is indeed an encryption for the password $p w$ and invalid otherwise. Note that also encryptions that are replayed by the adversary are oracle-generated.

Game 4. In this experiment all encryptions are oracle-generated and the simulator will keep a list for the projections $S_{i}$ he generated for each user $U_{i}$ in Round 1. Once an instance $\Pi_{i}^{s_{i}}$ has got all messages of the first round, the simulator checks if the received $S_{i-1}$ is consistent with the one generated for the $U_{i-1}$. In case $S_{i-1}$ was replaced and one of the respective $c_{i, i-1}$ or $c_{i-1, i}$ is oracle-generated, the corresponding key $Z_{i, i-1}$ is replaced by a random group element. If $Z_{i, i-1}$ was replaced, $U_{i-1}$ will use $X_{i-1,1}$ in Round 3.

The replacement of $Z_{i, i-1}$ was caused by a replaced projection $S_{i-1}$ and hence the value

$$
H_{k_{i-1}}\left(\text { label }_{\mathrm{i}, \mathrm{i}-1}, p w, c_{i, i-1}\right)
$$

may be known to the adversary. However, as $k_{i}$ was honestly generated, $c_{i-1, i}$ oracle-generated, and the projective hash function is smooth the hash $H_{k_{i}}\left(\right.$ label $\left._{-1, i}, p w, c_{i-1, i}\right)$ computed by $U_{i}$ is indistinguishable from an element chosen independently and uniformly in the group. Therefore $Z_{i, i-1}$ computed by $U_{i}$ is for the adversary indistinguishable from an independently uniformly at random chosen element. This holds of course only, if $U_{i-1}$ does not release any information about $H_{k_{i}}\left(\right.$ label $\left._{\mathrm{i}-1, \mathrm{i}}, p w, c_{i-1, i}\right)$. Therefore $U_{i-1}$ will use $X_{i-1,1}$ in Round 3 .

It is left to show that $U_{i-1}$ 's check of test ${ }_{i}$ will indeed fail. $U_{i}$ will randomly choose $\mathrm{UH}_{i}^{\prime} \in \mathcal{U H}^{\prime}$ and compute and broadcast test ${ }_{i}=\mathrm{UH}_{i}^{\prime}\left(Z_{i, i-1}\right)$. Neither can the adversary recognize the replacement of $Z_{i, i-1}$ from test ${ }_{i}$ nor can he produce a test $t_{i}$ that will be accepted by $U_{i-1}$.

- Even with knowledge of all test ${ }_{j}, j=1 \ldots n$, the value $Z_{i, i-1}$ remains indistinguishable from an independently and uniformly at random chosen element in $G$, because the test ${ }_{j}$ carry only a negligible amount of information due to $|T| \leq$ $\sqrt{|\mathbb{G}|}$.

- The adversary cannot produce test ${ }_{i}^{\prime}$ that would be accepted by $U_{i-1}$ : As $\mathrm{UH}_{i}^{\prime}$ is chosen independent at random, it is independent from $Z_{i-1, i}$ and the adversary has no prior information on the test $i_{i}$-value expected by $U_{i-1}$, because $Z_{i, i-1}$ is indistinguishable from random for him. Suppose the adversary tries to insert both test ${ }_{i}^{\prime}$ and $\mathrm{UH}_{i}^{\prime \prime}$. He will only succeed if he is able to find $h$ and $\mathrm{UH}_{i}^{\prime \prime}$ so that $h=\mathrm{UH}_{i}^{\prime \prime}\left(Z_{i-1, i}\right)$, where $Z_{i-1, i}$ is indistinguishable from random for him. By our assumption on the hash family $\mathcal{U} \mathcal{H}^{\prime}$ that there are no "bad indices" into $\mathcal{U H}^{\prime}$, this is not possible, however. Thus, in either case, the adversary has only a negligible probability of success.

Therefore, we have

$$
\left|\operatorname{Adv}\left(\mathcal{A}, G_{4}\right)-\operatorname{Adv}\left(\mathcal{A}, G_{3}\right)\right| \leq \operatorname{negl}(\ell) .
$$

Game 5. Again, the commitments are oracle-generated, but this experiment deviates from the previous one in that the simulator also checks if $S_{i+1}$ is consistent with the 
one generated for the $U_{i+1}$. In case $S_{i+1}$ was replaced and $c_{i, i+1}$ is oracle-generated, $U_{i}$ will continue with $X_{i, 1}$ in Round 3.

We show that $U_{i}$ 's check of test ${ }_{i+1}$ would indeed fail, so that this replacement makes no difference for the adversary. The argument is analogous as above: when $U_{i+1}$ chooses $\mathrm{UH}_{i+1}^{\prime} \in \mathcal{U} \mathcal{H}^{\prime}$, the adversary is unable to produce a test $t_{i+1}$ that will be accepted by $U_{i}$, as again the value $Z_{i, i+1}$ computed by $U_{i+1}$ is indistinguishable from random for the adversary. Neither will the adversary succeed in computing a pair $\left(\mathrm{UH}_{i+1}^{\prime \prime}\right.$, test $\left.{ }_{i+1}^{\prime}\right)$ that will convince $U_{i}$ : due to our assumption on the hash family $\mathcal{U H}^{\prime}$, the adversary has no a-priori information on the test ${ }_{i+1}$ value expected by $U_{i}$.

Therefore, we have

$$
\left|\operatorname{Adv}\left(\mathcal{A}, G_{5}\right)-\operatorname{Adv}\left(\mathcal{A}, G_{4}\right)\right| \leq \operatorname{negl}(\ell)
$$

Game 6. In this experiment, if the commitments were oracle-generated the simulator chooses the values $Z_{i, i-1}$ and $Z_{i, i+1}$ independently and uniformly at random from the group $G$. The simulator keeps a list for entries of the form

$$
\begin{aligned}
& \left(c_{i, i-1}, S_{i-1}, c_{i-1, i}, S_{i}\right) \rightarrow Z_{i, i-1}, \\
& \left(c_{i, i+1}, S_{i}, c_{i+1, i}, S_{i+1}\right) \rightarrow Z_{i, i+1} .
\end{aligned}
$$

The simulator behaves as in Game 5, except for the answer following a Send ${ }_{1}$ query that delivers the last first round message to an instance $\Pi_{i}^{s_{i}}$.

Once an instance $\Pi_{i}^{s_{i}}$ has received all messages of the first round, the simulator checks if $c_{i-1, i}$ and $c_{i+1, i}$ were both oracle-generated (but all projections were unmodified). In this case, the simulator checks if $\left(c_{i, i-1}, S_{i-1}, c_{i-1, i}, S_{i}\right) \rightarrow Z_{i, i-1}$ or $\left(c_{i, i+1}, S_{i}, c_{i+1, i}, S_{i+1}\right) \rightarrow Z_{i, i+1}$ are already in the list, and uses the according values $Z_{i, i-1}$ respectively $Z_{i, i+1}$ for further computations. The values $Z_{i, i-1}$ or $Z_{i, i+1}$ that are not yet determined by the list are chosen at random from the group $\mathbb{G}$ and the assignment $\left(c_{i, i-1}, S_{i-1}, c_{i-1, i}, S_{i}\right) \rightarrow Z_{i, i-1}$ or $\left(c_{i, i+1}, S_{i}, c_{i+1, i}, S_{i+1}\right) \rightarrow Z_{i, i+1}$, respectively, is stored in the list to assure consistency between neighbored instances.

Given an adversary $\mathcal{A}$ able to distinguish between Game 5 and Game 6 we can construct a distinguisher $D$ between $\operatorname{Exp}_{0}$ and $\operatorname{Exp}_{1}$. Thus, from Lemma 2 we can conclude that $\mathcal{A}$ 's advantage between the two games differs at most negligibly.

The distinguisher $D$ is either facing $\operatorname{Exp}_{0}$ or $\operatorname{Exp}_{1}$ from Lemma 2. $D$ is constructed so that it will behave like the simulator from Game 5, except the following:

- commitments $c$ are not computed but obtained by the $\mathcal{O}_{\text {Enc }}$ oracle from Lemma2 2 on input $p w$,
- if a Send-query of the adversary requires $D$ to compute values $Z_{i, i-1}$ respectively $Z_{i, i+1}, D$ will output hashes/random values from the respective values $c_{i, i-1}, c_{i-1, i}$ and $c_{i, i+1}, c_{i+1, i}$ if both were oracle-generated.

Now the view of $\mathcal{A}$ will be exactly as in Game 5 if $D$ is facing $\operatorname{Exp}_{0}$ and exactly as in Game 6 if $D$ is facing $\operatorname{Exp}_{1}$.

$$
\left|\operatorname{Adv}\left(\mathcal{A}, G_{6}\right)-\operatorname{Adv}\left(\mathcal{A}, G_{5}\right)\right| \leq \operatorname{negl}(\ell)
$$

For the following games, the simulator is given an Extract oracle, that checks if a given encryption is valid, i.e., an encryption of the password $p w$.

This can be done because the password is information-theoretically contained in the encryption. On input a value $c$, the Extract oracle exhausts all possible random choices $r$ to check whether $c$ is a commitment to $p w$ or not. Indeed, the set of possible values $r$ is of superpolynomial size in the security parameter; this is however allowed for the Extract oracle.

Game 7. In this experiment, the simulator behaves as in Game 6, except that in Round 2's computation phase, following a Send 1 query, the received $c_{i-1, i}$ and $c_{i+1, i}$ are checked by the simulator with respect to the password using the Extract oracle. Then, those instances $\Pi_{i}^{s_{i}}$ that received an invalid encryption will choose a random group element for $X_{i, 0}$.

By a statistical argument, we see that the probability for the adversary to distinguish between Game 7 and Game 6 is negligible. If in Round 1 an invalid encryption $c$ to a wrong password $\widetilde{p w}$ (that is, $(c, \widetilde{p w}) \notin L_{\rho}$,) was sent, then by the smoothness property of the hash proof system the distribution of $\left(c, \widetilde{p w}, \alpha(k), H_{k}(\right.$ label, $\left.\widetilde{p w}, c)\right)$ is statistically close to the distribution of $(c, \widetilde{p w}, \alpha(k), g)$ for a random group element $g \in G$. Thus, the respective $Z_{i, i-1}$ or $Z_{i, i+1}$ and therefore $X_{i, 0}$ will look like a random group element for the adversary, who thus has only a negligible chance to detect the difference.

As a result,

$$
\left|\operatorname{Adv}\left(\mathcal{A}, G_{7}\right)-\operatorname{Adv}\left(\mathcal{A}, G_{6}\right)\right| \leq \operatorname{negl}(\ell) .
$$

By now, only such executions of Round 2 following a Send $_{1}$ query are unchanged where the encryptions from the neighboring users are both valid and at least one was adversary-generated. The following experiments will also modify this situation.

Game 8. Now the simulator will abort the game with a win of the adversary, if an instance $\Pi_{i}^{s_{i}}$ received from a Send ${ }_{1}$-query valid commitments $c_{i-1, i}$ and $c_{i+1, i}$ of which at least one was adversary-generated. 
This will only increase the success probability of the adversary, therefore:

$$
\operatorname{Adv}\left(\mathcal{A}, G_{8}\right) \geq \operatorname{Adv}\left(\mathcal{A}, G_{7}\right)
$$

Game 9. In this game, the simulation aborts if the adversary has inserted commitments, projections or $X_{i}$-values in Round 3:

Note that in Game 9, if a message in Round 1 to an instance of $U_{i}$ was modified, as a result $U_{i}$ individually chooses $Z_{i, i-1}$ or $Z_{i, i+1}$, respectively, uniformly at random. Therefore, $U_{i}$ holds $X_{i}$ unknown to anyone and expects commitments to values $X_{j}$ such that $X_{1} \cdots X_{n}=1$. Now, in Round 2, $U_{i}$ outputs a commitment $C_{\rho}\left(U_{i}, X_{i} ; r_{i}^{\prime}\right)$ to a value $X_{i}$ that only with negligible probability fulfills $X_{1} \cdots X_{n}=1$. To avoid users $U_{1}, \ldots, U_{i-1}, U_{i+1}, \ldots, U_{n}$ from aborting, therefore, the adversary needs to be able to construct a commitment $C_{\rho}\left(U_{i}, X_{i}^{*} ; r^{*}\right)$ to a value $X_{i}^{*}$ such that $X_{1} \cdots X_{i}^{*} \cdots X_{n}=1$. Again, as all $X_{j}$ are unknown to the adversary, this can only succeed with negligible probability. Note that, moreover, $X_{i}$ is a random value and only $C_{\rho}\left(U_{i}, X_{i} ; r_{i}^{\prime}\right)$ contains information about $X_{i}$. Thus, the non-malleability of the commitment scheme gives the adversary only a negligible probability to insert values $X_{j}^{*}$ with $j=1, \ldots, i-1, i+1, \ldots, n$ which would be accepted by $\Pi_{i}^{s_{i}}$ in Game 8 . The above argument also demonstrates that the adversary cannot insert any value $X_{i}$ in Round 3 without resulting in an abort (with overwhelming probability). Therefore,

$$
\left|\operatorname{Adv}\left(\mathcal{A}, G_{9}\right)-\operatorname{Adv}\left(\mathcal{A}, G_{8}\right)\right| \leq \operatorname{negl}(\ell) .
$$

At this point, we have excluded all situations in which the adversary may have inserted encryptions, commitments, projections or $X_{i}$-values in Round 3: either he has guessed the password and inserted valid commitments to it (Game 8) or his attempts to insert rogue messages resulted in a protocol abortion before the computation of a session key. Thus the only situation left to handle is that all queries to the Send-oracle contain messages faithfully constructed following the protocol specification. Here we can mimic the reasoning for the passive case.

Game 10. Now the simulation changes in that, for constructing the encryptions from Round 1, a password $\widehat{p w}$ is chosen uniformly at random from $\mathcal{D}$. This does not change anything, as in the previous game, these values were not used in any projective hash function anymore. All information about the password, which was available to the adversary, were encryptions sent in the first round. Due to the semantic security of the encryption scheme, the adversary detects the use of $\widehat{p w}$ instead of $p w$ with a negligible probability only. Now the adversary does not have any correct encryption of the password as input.
But, due to the non-malleability of the encryption scheme, the adversary's probability to succeed in producing a new valid encryption of $p w$ drops at most negligibly,

$$
\left|\operatorname{Adv}\left(\mathcal{A}, G_{10}\right)-\operatorname{Adv}\left(\mathcal{A}, G_{9}\right)\right| \leq \operatorname{negl}(\ell) .
$$

Game 11. We now modify the computation of the session key. The simulator keeps a list of assignments $\left(Z_{1,2}, \ldots, Z_{n, 1}, \mathrm{sk}_{i}^{s_{i}}\right)$. Once an instance receives the last Send $_{3}$-query, the simulator computes $Z_{1,2}, \ldots, Z_{n, 1}$ and checks if for the sequence $\left(Z_{1,2}, \ldots, Z_{n, 1}\right)$ a master key was already issued and assigns this key to the instance. If no such entry exists in the list, the simulator chooses a session key $\mathrm{sk}_{i}^{s_{i}} \in\{0,1\}^{\ell}$ uniformly at random.

The adversary can only detect the difference, if he knows the master key $\mathrm{mk}=\left(Z_{1,2}, \ldots, Z_{n, 1}\right)$. The master key has once the $X_{i}$ are public, sufficient entropy because knowing all quotients $X_{i}$, still the value of at least one of the two-party keys $Z_{k, j}$ is indistinguishable from a random group element. Therefore the output of the function $F_{\mathrm{UH}(\mathrm{mk})}$ is only with a negligible probability distinguishable from a random $\mathrm{sk}_{i}^{s_{i}}$,

$$
\left|\operatorname{Adv}\left(\mathcal{A}, G_{11}\right)-\operatorname{Adv}\left(\mathcal{A}, G_{10}\right)\right| \leq \operatorname{negl}(\ell)
$$

Now the session keys are randomly distributed and independent from the password and the messages. Instances that hold the same master key computed the same $\mathrm{UH}(\mathrm{mk})$ and therefore hold identical session identifiers. Thus, those instances are partnered and the freshness definition renders the Reveal-oracle useless because instances that are not partnered have independently uniformly at random chosen session keys. Besides the $1 / 2$ probability of guessing the bit $b$ right, the only way for the adversary to win is having sent a valid adversary-generated encryption to a neighbored instance that did not get an invalid commitment from the other neighbor. Thus, the adversary has just one try per instance to guess a password and the probability to win in Game 11 is

$$
\operatorname{Succ}\left(\mathcal{A}, G_{11}\right)=\frac{q}{|\mathcal{D}|}+\frac{1}{2}\left(1-\frac{q}{|\mathcal{D}|}\right)+\operatorname{negl}(\ell)
$$

giving an advantage of

$$
\operatorname{Adv}\left(\mathcal{A}, G_{11}\right)=\frac{q}{|\mathcal{D}|}+\operatorname{negl}(\ell)
$$

Remember, that $q$ only counts the number of different instances that were addressed by a Send-query.

Putting everything together, we have

$$
\operatorname{Adv}(\mathcal{A}) \leq \frac{q}{|\mathcal{D}|}+\operatorname{negl}(\ell)
$$




\section{Conclusion}

We describe a generic design for group PAKE building on the two-party protocol of Katz and Vaikuntanathan (2013). Following Ben Hamouda et al. (2013), this scheme can be implemented from the Cramer-Shoup CCA-encryption, resulting in a very efficient (pairing free) design. Our construction can be proven secure in the standard model, and provides quite strong guarantees; in particular, we evidence that adversaries can only perform one online password test per instance.

\section{Acknowledgment}

M.I. González Vasco and R. Steinwandt are partially supported by research projects MTM2013-41426-R and MTM2016-77213-R, both funded by the Spanish MINECO. Also, this research was sponsored (in part) by the NATO Science for Peace and Security Programme under the grant G5448. We are indebted to Michel Abdalla for numerous valuable comments and discussions.

\section{References}

Abdalla, M., Benhamouda, F. and MacKenzie, P. (2015). Security of the J-PAKE password-authenticated key exchange protocol, IEEE Symposium on Security and Privacy, SP 2015, San Jose, CA, USA, pp. 571-587.

Abdalla, M., Bohli, J.-M., González Vasco, M.I. and Steinwandt, R. (2007). (Password) Authenticated key establishment: From 2-party to group, in S.P. Vadhan (Ed.), Theory of Cryptography Conference, TCC 2007, Lecture Notes in Computer Science, Vol. 4392, Springer, Berlin/Heidelberg, pp. 499-514.

Abdalla, M., Bresson, E., Chevassut, O. and Pointcheval, D. (2006). Password-based group key exchange in a constant number of rounds, in M. Yung et al. (Eds), Public Key Cryptography, PKC 2006, Lecture Notes in Computer Science, Vol. 3958, Springer, Berlin/Heidelberg, pp. 427-442.

Abdalla, M., Fouque, P.-A. and Pointcheval, D. (2005). Password-based authenticated key exchange in the three-party setting, in S. Vaudenay (Ed.), Public Key Cryptography, PKC 2005, Lecture Notes in Computer Science, Vol. 3386, Springer, Berlin/Heidelberg, pp. 65-84.

Abdalla, M., Fouque, P.-A. and Pointcheval, D. (2006). Password-based authenticated key exchange in the three-party setting, IEE Proceedings: Information Security 153(1): 27-39.

Abdalla, M. and Pointcheval, D. (2005). Simple password-based encrypted key exchange protocols, in A. Menezes (Ed.), Topics in Cryptology, CT-RSA 2005, Lecture Notes in Computer Science, Vol. 3376, Springer, Berlin/Heidelberg, pp. 191-208.

Abdalla, M. and Pointcheval, D. (2006). A scalable password-based group key exchange protocol in the standard model, in X. Lai and K. Chen (Eds), Proceedings of ASIACRYPT 2006, Lecture Notes in Computer Science, Vol. 4284, Springer, Berlin/Heidelberg, pp. 332-347.

Bellare, M., Canetti, R. and Krawczyk, H. (1998). A modular approach to the design and analysis of authentication and key exchange protocols (extended abstract), 13th Annual ACM Symposium on the Theory of Computing, Dallas, TX, USA, pp. 419-428, DOI: 10.1145/276698.276854.

Bellare, M., Pointcheval, D. and Rogaway, P. (2000). Authenticated key exchange secure against dictionary attacks, in B. Preneel (Ed.), Advances in Cryptology, EUROCRYPT 2000, Lecture Notes in Computer Science, Vol. 1807, Springer, Berlin/Heidelberg, pp. 139-155.

Bellare, M. and Rogaway, P. (1994). Entity authentication and key distribution, in D.R. Stinson (Ed.), Advances in Cryptology, CRYPTO'93, Lecture Notes in Computer Science, Vol. 773, Springer, Berlin/Heidelberg, pp. 232-249.

Ben Hamouda, F., Blazy, O., Chevalier, C., Pointcheval, D. and Vergnaud, D. (2013). New smooth projective hash functions and one-round authenticated key exchange, IACR Cryptology ePrint Archive 2013: 34, http: / / eprint. iacr.org/2013/034

Blake-Wilson, S. and Menezes, A. (1999). Authenticated Diffie-Hellman key agreement protocols, in S.E. Tavares and H. Meijer (Eds), Proceedings of the Selected Areas in Cryptography, SAC'98, Springer-Verlag, Berlin/Heidelberg, pp. 339-361.

Blazy, O. and Chevalier, C. (2015). Generic construction of UC-secure oblivious transfer, in T. Malkin et al. (Eds), Applied Cryptography and Network Security, Lecture Notes in Computer Science, Vol. 9092, Springer, Berlin/Heidelberg, pp. 65-86.

Bohli, J.-M., González Vasco, M.I. and Steinwandt, R. (2007). Secure group key establishment revisited, International Journal of Information Security 6(4): 243-254.

Bohli, J.-M., Vasco, M.I.G. and Steinwandt, R. (2018). Password-authenticated constant-round group key establishment from smooth projective hash functions, Cryptology ePrint Archive, Report 2006/214, http: / / e print.iacr.org/2006/214

Boyko, V., MacKenzie, P. and Patel, S. (2000). Provably secure password-authenticated key exchange using Diffie-Hellman, in B. Preneel (Ed.), Advances in Cryptology, EUROCRYPT 2000, Lecture Notes in Computer Science, Vol. 1807, Springer, Berlin/Heidelberg, pp. 156-171.

Bresson, E., Chevassut, O. and Pointcheval, D. (2002). Group Diffie-Hellman key exchange secure against dictionary attacks, in Y. Zheng (Ed.) Advances in Cryptology, Lecture Notes in Computer Science, Vol. 2501, Springer, Berlin/Heidelberg, pp. 497-514.

Burmester, M. and Desmedt, Y. (1995). A secure and efficient conference key distribution system, in A.D. Santis (Ed.), Advances in Cryptology, EUROCRYPT'94, Lecture Notes in Computer Science, Vol. 950, Springer, Berlin/Heidelberg, pp. 275-286. 
Carter, L. and Wegman, M.N. (1977). Universal classes of hash functions (extended abstract), in J.E. Hopcroft et al. (Eds), Proceedings of the 9th Annual ACM Symposium on Theory of Computing, Boulder, CO, USA, pp. 106-112.

Cramer, R. and Shoup, V. (2002). Universal hash proofs and a paradigm for adaptive chosen ciphertext secure public-key encryption, in L. Knudsen (Ed.), Advances in Cryptology, EUROCRYPT 2002, Lecture Notes in Computer Science, Vol. 2332, Springer, Berlin/Heidelberg, pp. 45-64.

Dutta, R. and Barua, R. (2006). Password-based encrypted group key agreement, International Journal of Network Security 3(1): 23-34.

Gennaro, R. and Lindell, Y. (2003a). A framework for password-based authenticated key exchange, Cryptology ePrint Archive, Report 2003/032, http: //eprint.ia Cr.org/2003/032

Gennaro, R. and Lindell, Y. (2003b). A framework for password-based authenticated key exchange (extended abstract), in E. Biham (Ed.), Advances in Cryptology, EUROCRYPT 2003, Lecture Notes in Computer Science, Vol. 2656, Springer, Berlin/Heidelberg, pp. 524-543.

Gennaro, R. and Lindell, Y. (2006). A framework for password-based authenticated key exchange, ACM Transactions on Information and System Security 9(2): 181-234, DOI: $10.1145 / 1151414.1151418$.

González Vasco, M.I., Martínez, C., Steinwandt, R. and Villar, J.L. (2005). A new Cramer-Shoup like methodology for group based provably secure schemes, in J. Kilian (Ed.), Proceedings of the 2nd Conference on Theory of Cryptography, TCC 2005, Lecture Notes in Computer Science, Vol. 3378, Springer, Berlin/Heidelberg, pp. 495-509.

Gorantla, M.C., Boyd, C., González Nieto, J.M. and Manulis, M. (2010). Generic one round group key exchange in the standard model, Information, Security and Cryptology, ICISC 2009, Lecture Notes in Computer Science, Vol. 5984, Springer, Berlin/Heidelberg, pp. 1-15.

Hwang, J.Y., Lee, S.-M. and Lee, D.H. (2004). Scalable key exchange transformation: From two-party to group, Electronic Letters 40(12): 728-729.

Kalai, Y.T. (2005). Smooth projective hashing and two-message oblivious transfer, in R. Cramer (Ed.), Advances in Cryptology, EUROCRYPT 2005, Lecture Notes in Computer Science, Vol. 3494, Springer, Berlin/Heidelberg, pp. 78-95.

Katz, J., Ostrovsky, R. and Yung, M. (2001). Efficient password-authenticated key exchange using human-memorable passwords, in B. Pfitzmann (Ed.), Advances in Cryptology, EUROCRYPT 2001, Lecture Notes in Computer Science, Vol. 2045, Springer, Berlin/Heidelberg, pp. 475-494.

Katz, J., Ostrovsky, R. and Yung, M. (2006). Efficient and secure authenticated key exchange using weak passwords, http://www.cs.umd.edu/ jkatz/pa pers/password.pdf
Katz, J. and Shin, J.S. (2005). Modeling insider attacks on group key-exchange protocols, Cryptology ePrint Archive, Report 2005/163, http://eprint.iacr.org/2005 1163.

Katz, J. and Vaikuntanathan, V. (2013). Round-optimal password-based authenticated key exchange, Journal of Cryptology 26(4): 714-743.

Katz, J. and Yung, M. (2007). Scalable protocols for authenticated group key exchange, Journal of Cryptology 20(1): $85-113$

Kurosawa, K. and Desmedt, Y. (2004). A new paradigm of hybrid encryption scheme, in M. Franklin (Ed.), Advances in Cryptology, CRYPTO 2004, Lecture Notes in Computer Science, Vol. 3152, Springer, Berlin/Heidelberg, pp. 426-442.

Mayer, A. and Yung, M. (1999). Secure protocol transformation via "Expansion": From two-party to groups, Proceedings of the 6th ACM Conference on Computer and Communications Security, CCS'99, New York, NY, USA, pp. 83-92.

Nam, J., Paik, J. and Won, D. (2011). A security weakness in Abdalla et al.'s generic construction of a group key exchange protocol, Information Sciences 181(1): 234-238, DOI: 10.1016/j.ins.2010.09.011.

Shoup, V. (2006). An emerging standard for public-key encryption, ISO 18033-2, International Organization for Standardization, Geneva, http: / / www . shoup. net/i so/sta6.paf

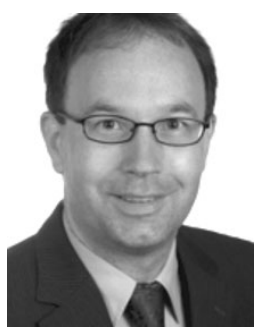

Jens-Matthias Bohli is a professor at the University of Applied Science in Mannheim. He received his diploma and the $\mathrm{PhD}$ degree in computer science from the Karlsruhe Institute for Technology in 2003 and 2007, respectively. In 2007, he joined the security research team at NEC Laboratories Europe as a senior researcher and worked on security for the IoT. In the academic year 2009/2010, he worked at the University of Sussex as a lecturer. In 2016, he joined the University of Applied Science in Mannheim. His current research interest and experience lie in efficient cryptographic protocols for a wide range of applications, such as authentication, blockchains, voting machines, and embedded systems.

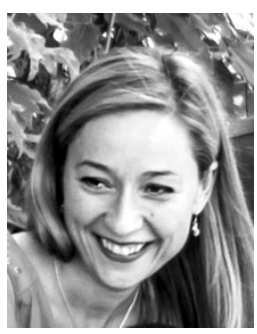

María Isabel González Vasco is an associate professor at the Department of MACIMTE, King Juan Carlos University, where she has been working since 2003. She received her diploma and the $\mathrm{PhD}$ degree in mathematics from the University of Oviedo (1999 and 2003). Her research interests include provable security for cryptographic constructions, with a special focus on public key cryptographic designs for encryption and group key exchange. She is currently a member of the board of directors (Junta de Gobierno) of the Royal Spanish Mathematical Society. 


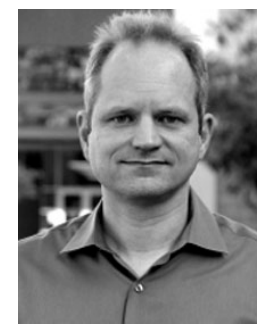

mation Security.

Rainer Steinwandt serves as the chair of Florida

Atlantic University's Department of Mathemati-

cal Sciences. Before joining FAU, he was with

the University of Karlsruhe in Germany, where

he completed his $\mathrm{MS}$ and $\mathrm{PhD}$ degrees in com-

puter science, researching topics in computer al-

gebra. Today, his research focus is on cryptology,

including quantum cryptanalysis and quantum-

safe cryptography. He currently serves as the director of FAU's Center for Cryptology and Infor-

Received: 1 November 2018

Revised: 4 March 2019

Accepted: 8 May 2019 